\title{
Pharmacological studies of Pediococcus on performance and some hematological and biochemical parameters in cattle
}

\author{
El-Sayed, M. G.*, Mohamed El-Diasty **and Shimaa W. Zayed** \\ *Department of Pharmacology Faculty of Vet. Med. Mansoura University. Egypt \\ ** Animal Health Research Institute, Mansoura Provincial Lab., Egypt
}

\section{ABSTRACT}

\begin{abstract}
The use of Pediococcus $\left(23 \times 10^{6} \mathrm{CFU} / \mathrm{calf} /\right.$ day) orally in drinking milk once daily for one month in Holstein calves have highly significant effect on blood picture, liver function tests, kidney function test ,blood electrolytes and body weight in cow calves. There were a significant increase in total erythrocytes count, hemoglobin content, packed cell volume, mean corpuscular volume, mean corpuscular hemoglobin, mean corpuscular hemoglobin concentration, thrombocyte count, total leucocytes count, neutrophils counts, eosinophil, basophils, total protein and its fractions, level of alkaline phosphatase (ALP),level of sodium ,calcium , phosphorus and body weight. On the other hand there were significant decrease in urea and potassium level in groups treated by Pediococcus compared to the control groups. Moreover there were a non-significant changes in lymphocytes, monocytes, creatinine, alanine transaminase (ALT), aspartate transaminase (AST), in the same groups compared to the control groups.
\end{abstract}

\section{INTRODUCTION}

In the light of population inflation in Egypt and increase the need for animal protein and milk and keep pace with global trends of the use of probiotics to improve the overall health of the animal, resulting in increased productivity and reproductive efficiency, we start our research plan.

Numerous studies have been conducted in an attempt to increase ruminant productivity by manipulating the rumen environment and to increase feed digestibility and nutrient utilization by the animals in order to supply sufficient nutrients to support a high level of milk production. One approach that has recently been widely investigated is the application of direct-fed microbial (DFM) preparations, in order to promote digestion and intestinal hygiene, enhance animal performance and reduce usage of antibiotics. The definition of DFM is very broad and may include specific and nonspecific yeast, fungi, bacteria, cell fragments, and filtrates. (Sobhy et al., 2014).

Probiotics, which are live cultures of harmless bacteria or yeast species that equilibrate intestinal micro flora to benefit the host (Fuller, 1989), have been demonstrated to be useful in maintaining the intestinal ecosystem and improving animal health. Because of a ban on the use of antibiotics, probiotics have been suggested as the most desirable alternative for livestock due to their beneficial effects. (Meng et al., 2010(
The most commonly used organisms in probiotic preparations are the lactic acid bacteria (Lactobacillus, Streptococci, bifidobacteria and Pediococcus).These are found in large numbers in the gut of healthyanimals and do not appear to affect them adversely. Vibhute et al., (2011(

Pediococcus acidilactici and Pediococcus Pentosaceus are a species of Gram-positive cocci which prevent colonization of pathogens which has great effect to treat digestive disorders. Pediococcus exert antagonism against other microorganisms, including enteric pathogen through the production of lactic acid and secretion of bacteriocins known as pediocins. .Pediococcus stimulates humoral immune response against infectious parasitic pathogens by possible competitive inhibition and pediocins production, which inhibit pathogenic bacteria and other Gram-positive spoilage. Haakensen et al., (2009)

This work was planned to evaluate some pharmacological effects of Pediococcus on hematological parameters, some liver and kidney functions, level of some electrolytes and on body weight gain in cow calves.

\section{MATERIALS AND METHODS}

1. Drugs: Max Boost powder Plus Sachet 100 gm. Powder prepared for oral administration contain:

$$
\text { -Pediococcus Acidilactici } 1 \times 10^{6} \mathrm{cfu} / \mathrm{g}
$$


-Pediococcus Pentosaceus $1.3 \times 10^{6} \mathrm{cfu} / \mathrm{g}$

-Dextrose Carrier

Manufactured \&Exported by DVS Biolife limited - India

Elcon Nova-43 Degla Street-Mohandesen-Giza

Email:info@elconnova.com Reg. No: 5076 in 27/3/2013

Dose: $10 \mathrm{gm}$. / head / daily for 30 days orally that give $23 \times 10^{6}$ cfu /10 gm. (Frizzo et al., 2010)

(CFU=COLONY FORMING UNITS)

2. Experimental calves:

Twenty Holstein calves at Damietta governorate in special dairy farm were divided into four groups $(5$ male and 5 females as a control group) and ( 5 male and 5 female under our experiment); all groups under the same hygiene management and the same nutrition (starter ration $21 \%$ - AlAsema Group Company + milk before weaning while after weaning the ration were be $18 \%$ until reach $200-220 \mathrm{~kg}$ b.wt then $16 \%$ ration till reach moreover $350 \mathrm{~kg}$ b.wt. from the same company). Every calves under our experiment (10 calves) were take $10 \mathrm{gm}$. Maxboost* after fermentation in 100 $\mathrm{ml}$ water for $2-3$ hours then added to the milk one time per day for one month, then after 1 month give the drug for 3 successive days $20 \mathrm{gm} / \mathrm{head} /$ once daily in drinking water after fermentation for 2-3 hours for five months. Body weight were determined in the beginning of the experiment and every week for 4 weeks then every 2 months for 10 months to the all groups, moreover the observation of the general health conditions.

\section{Sampling}

Two blood samples (the first sample for hematological studies and the second sample for serological studies) were collected from each animal in the four groups at zero day and after 15 days, 30 days and 45 days post the drugs administration, Stoffregen et al., 1997.

4. Hematological studies:

A-Mindray BC-2800:

Mindray Auto Hematology Analyzer BC 2800 is UN automated hematology analyzer designed for in vitro diagnostics use in blood cell counting and characterization.

\section{RESULTS}

-1 The effect of oral administration of Pediococcus $(23 \times 106$ CFU/animal) in drinking milk once daily for one month on total erythrocytes count, hemoglobin content and packed cell volume in cow calves as shown in Table (1.)(

-2 The effect of oral administration of Pediococcus $(23 \times 106$ CFU/animal) in drinking milk once daily for one month on some blood picture in cow calves as shown in Table (2.)(

-3 The effect of oral administration of Pediococcus (23×106 CFU/animal) in drinking milk once daily for one month on total and differential leucocytes counts in cow calves as shown in Table (3.)
B-Differential leukocyte count (thousand $/ \mathrm{mm}^{3}$ ): according to Feldman et al., (2000).

C- Serum Biochemical Analysis:

Biochemical serum analysis of total proteins, albumin, ALT, AST, ALP, Urea, creatinine, sodium, potassium, calcium and phosphorus.

(a)Liver function tests:

I-Determination of Total Protein: according to the method of (Doumas, 1975)

II-Determination of Albumin: according to Doumas et al., 1981

III-Serum globulin calculation: as described by Doumas and Biggs, 1972

IV-Determination of serum Transaminases (ALT) and (AST): according to Reitman and Frankel, 1957.

VI-Determination of serum alkaline phosphatase (ALP) according to Rosalki (1993)

(b)Kidney function tests:

I-Determination of serum Creatinine was determined calorimetrically according toHenry (1974)

II-Determination of Urea: was performed according to the method of Patton and Crouch 1977.

(c) Determination of electrolytes:

I-determination of serum Calcium: according to the method of Gindler and King, 1972.

II-Determination of serum phosphorus: according to the method of El-Merzabani, 1977.

III- Determination of serum sodium: according to the method of Henry et al., 1974.

IV-Determination of serum Potassium: according to the method Turbid metric Tetraphenylborate (TPB), Henry et al., 1974.

5. Statistical analysis as described by one way (ANOVA), using SPSS computer program (version. 20). (SPSS, 2015)

-4 The effect of oral administration of Pediococcus (23×106 CFU/animal) in drinking milk once daily for one month on creatinine and urea in cow calves as shown in Table (4)

$-5 \quad$ The effect of oral administration of Pediococcus (23×106 CFU/animal) in drinking milk once daily for one month on total protein and its fractions in cow calves as shown in Table (5.)(

-6 The effect of oral administration of Pediococcus (23×106 CFU/animal) in drinking milk once daily for one month on alanine transaminase (ALT), aspartate transaminase (AST) and alkaline phosphatase (ALP) in cow calves as shown in Table (6.)(

$-7 \quad$ The effect of oral administration of Pediococcus (23×106 CFU/animal) in drinking milk once daily for one month on blood electrolytes in cow calves as shown in Table (7.)( 
-8 The effect of oral administration of Pediococcus

on body weight in cow calves as shown in Table (8).

(23×106 CFU/animal) in drinking milk once daily for one month

Table (1): The effect of oral administration of Pediococcus $\left(23 \times 10^{6} \mathrm{CFU} /\right.$ animal) in drinking milk once daily for one month on some blood picture in cow calves.

\begin{tabular}{|c|c|c|c|c|c|c|c|c|c|c|c|c|}
\hline \multirow{3}{*}{ Group } & \multicolumn{4}{|c|}{$\operatorname{RBCs}\left(\mathrm{n} \times 10^{6} / \mathrm{ML}\right)$} & \multicolumn{4}{|c|}{$\mathrm{Hb}(\mathrm{gm} . / \mathrm{dl})$} & \multicolumn{4}{|c|}{ PCV (\%) } \\
\hline & \multicolumn{4}{|c|}{ Time (Week) } & \multicolumn{4}{|c|}{ Time (Week) } & \multicolumn{4}{|c|}{ Time (Week) } \\
\hline & 0 & $2^{\text {nd }} w$ & $4^{\text {th }} w$ & $6^{\text {th }} w$ & 0 & $2^{\text {nd }} w$ & $4^{\text {th }} w$ & $6^{\text {th }} w$ & 0 & $2^{\text {nd }} w$ & $4^{\text {th }} w$ & $6^{\text {th }} w$ \\
\hline $\begin{array}{l}\text { Control } \\
\text { male }\end{array}$ & $\begin{array}{l}5.82 \\
\pm 0.54^{b}\end{array}$ & $\begin{array}{l}4.74 \pm \\
0.45^{a}\end{array}$ & $\begin{array}{l}4.82 \pm . \\
45^{\mathrm{b}}\end{array}$ & $\begin{array}{l}5.50 \pm \\
0.46^{c}\end{array}$ & $\begin{array}{l}7.24 \pm 0 . \\
06^{\mathrm{bc}}\end{array}$ & $\begin{array}{l}7.00 \pm 0 . \\
45^{\mathrm{a}}\end{array}$ & $\begin{array}{l}8.66 \pm 0 . \\
27^{\mathrm{a}}\end{array}$ & $\begin{array}{l}8.14 \pm 0 . \\
20^{\mathrm{a}}\end{array}$ & $\begin{array}{l}22.30 \\
\pm 0.58^{b}\end{array}$ & $\begin{array}{l}22.88 \\
\pm 0.51^{\text {a }}\end{array}$ & $\begin{array}{l}22.36 \pm 0 \\
.63^{b}\end{array}$ & $\begin{array}{l}23.43 \pm 0 . \\
68^{\mathrm{ab}}\end{array}$ \\
\hline $\begin{array}{l}\text { Treated } \\
\text { male }\end{array}$ & $\begin{array}{l}7.43 \pm 0 . \\
32^{\mathrm{a}}\end{array}$ & $\begin{array}{l}6.69 \pm \\
0.34^{b}\end{array}$ & $\begin{array}{l}7.63 \pm \\
0.41^{a}\end{array}$ & $\begin{array}{l}7.47 \pm \\
0.49^{\mathrm{ab}}\end{array}$ & $\begin{array}{l}7.92 \pm 0 . \\
50^{\mathrm{ab}}\end{array}$ & $\begin{array}{l}7.36 \pm 0 . \\
50^{\mathrm{a}}\end{array}$ & $\begin{array}{l}8.12 \pm 0 . \\
60^{\mathrm{ab}}\end{array}$ & $\begin{array}{l}7.60 \pm 0 . \\
50^{\mathrm{a}}\end{array}$ & $\begin{array}{l}25.34 \\
\pm 1.73^{\mathrm{a}} \\
\mathrm{b}\end{array}$ & $\begin{array}{l}22.10 \\
\pm 1.54^{\mathrm{a}}\end{array}$ & $\begin{array}{l}24.17 \pm 2 \\
.05^{\mathrm{ab}}\end{array}$ & $\begin{array}{l}25.40 \pm 2 . \\
04^{\mathrm{ab}}\end{array}$ \\
\hline $\begin{array}{l}\text { Control } \\
\text { female }\end{array}$ & $\begin{array}{l}5.52 \pm 0 . \\
32^{\mathrm{b}}\end{array}$ & $\begin{array}{l}4.96 \pm \\
0.35^{\mathrm{a}}\end{array}$ & $\begin{array}{l}5.21 \pm \\
0.73^{b}\end{array}$ & $\begin{array}{l}6.20 \pm \\
0.49^{b c}\end{array}$ & $\begin{array}{l}6.76 \pm 0 . \\
27^{c}\end{array}$ & $\begin{array}{l}7.72 \pm 0 . \\
13^{\mathrm{a}}\end{array}$ & $\begin{array}{l}7.30 \pm 0 . \\
51^{\mathrm{b}}\end{array}$ & $\begin{array}{l}7.14 \pm 0 . \\
17^{\mathrm{a}}\end{array}$ & $\begin{array}{l}25.42 \\
\pm 0.63^{a}\end{array}$ & $\begin{array}{l}23.92 \\
\pm 0.63^{a}\end{array}$ & $\begin{array}{l}24.16 \pm 0 \\
.43^{\text {ab }}\end{array}$ & $\begin{array}{l}22.20 \pm 0 . \\
40^{\mathrm{b}}\end{array}$ \\
\hline $\begin{array}{l}\text { Treated } \\
\text { female }\end{array}$ & $\begin{array}{l}7.75 \pm 0 . \\
24^{\mathrm{a}}\end{array}$ & $\begin{array}{l}7.08 \pm \\
0.18^{b}\end{array}$ & $\begin{array}{l}7.65 \pm \\
0.22^{\mathrm{a}}\end{array}$ & $\begin{array}{l}7.72 \pm \\
0.43^{\mathrm{a}}\end{array}$ & $\begin{array}{l}8.64 \pm 0 . \\
33^{\mathrm{a}}\end{array}$ & $\begin{array}{l}7.86 \pm 0 . \\
27^{\mathrm{a}}\end{array}$ & $\begin{array}{l}8.26 \pm 0 . \\
20^{\mathrm{ab}}\end{array}$ & $\begin{array}{l}7.84 \pm 0.4 \\
0^{\mathrm{a}}\end{array}$ & $\begin{array}{l}27.48 \\
\pm 1.78^{\mathrm{a}}\end{array}$ & $\begin{array}{l}24.28 \\
\pm 1.04^{\mathrm{a}}\end{array}$ & $\begin{array}{l}26.64 \pm 1 \\
.26^{\mathrm{a}}\end{array}$ & $\begin{array}{l}27.32 \pm 1 . \\
85^{\mathrm{a}}\end{array}$ \\
\hline
\end{tabular}

The different litters in the same column mean significance at $(p<0.05)$.

(Mean \pm S.E) $\quad n=5$

Table (2): The effect of oral administration of Pediococcus $\left(23 \times 10^{6} \mathrm{CFU} /\right.$ animal) in drinking milk once daily for one month on some blood picture in cow calves.

\begin{tabular}{|c|c|c|c|c|c|}
\hline Items & Time (Week) & Control male & Treated male & Control female & Treated female \\
\hline \multirow{4}{*}{$\operatorname{MCV}(\mathrm{fl})$} & 0 & $22.57 \pm 0.55^{b}$ & $34.02 \pm 0.90^{\mathrm{a}}$ & $25.72 \pm 1.88^{b}$ & $35.42 \pm 1.49^{\mathrm{a}}$ \\
\hline & $2 w$ & $23.90 \pm .24^{b}$ & $33.00 \pm 0.76^{a}$ & $24.25 \pm 0.32^{b}$ & $34.34 \pm 0.93^{\mathrm{a}}$ \\
\hline & $4 w$ & $27.04 \pm 1.83^{b}$ & $32.82 \pm 0.95^{\mathrm{a}}$ & $.60 \pm 1.07^{b}$ & $34.82 \pm 0.94^{\mathrm{a}}$ \\
\hline & $6 w$ & $26.80 \pm 1.24^{b}$ & $33.94 \pm 0.68^{\mathrm{a}}$ & $30.20 \pm 1.56^{b}$ & $35.40 \pm 0.96^{\mathrm{a}}$ \\
\hline \multirow{5}{*}{$\mathrm{MCH}$ (pg.) } & 0 & $8.40 \pm 0.92^{b}$ & $10.78 \pm 0.25^{\mathrm{a}}$ & $6.80 \pm 0.37^{c}$ & $11.08 \pm 0.20^{\mathrm{a}}$ \\
\hline & $2 w$ & $7.00 \pm 0.45^{b}$ & $10.92 \pm 0.32^{\mathrm{a}}$ & $6.80 \pm 0.58^{b}$ & $11.04 \pm 0.18^{\mathrm{a}}$ \\
\hline & $4 w$ & $6.20 \pm 0.58^{b}$ & $10.10 \pm 0.21^{\mathrm{a}}$ & $7.80 \pm 0.37^{b}$ & $10.76 \pm 0.17^{\mathrm{a}}$ \\
\hline & $6 w$ & $7.80 \pm 0.37^{b}$ & $10.10 \pm 0.25^{a}$ & $7.80 \pm 0.37^{b}$ & $10.10 \pm 0.17^{\mathrm{a}}$ \\
\hline & 0 & $27.00 \pm 0.70^{c}$ & $31.92 \pm 1.17^{\mathrm{a}}$ & $27.20 \pm 0.66^{\mathrm{bc}}$ & $29.84 \pm 1.43^{\mathrm{ab}}$ \\
\hline \multirow[t]{4}{*}{$\mathrm{MCHC}(\%)$} & $2 w$ & $26.00 \pm 0.70^{b}$ & $33.32 \pm 1.21^{\mathrm{a}}$ & $25.80 \pm 0.92^{b}$ & $32.44 \pm 1.06^{\mathrm{a}}$ \\
\hline & $4 w$ & $25.20 \pm 0.73^{c}$ & $30.22 \pm 0.37^{\mathrm{a}}$ & $28.20 \pm 0.37^{b}$ & $31.12 \pm 0.84^{\mathrm{a}}$ \\
\hline & $6 w$ & $25.20 \pm 0.97^{b}$ & $30.06 \pm 0.95^{\mathrm{a}}$ & $25.60 \pm 0.60^{b}$ & $28.72 \pm 0.35^{\mathrm{a}}$ \\
\hline & 0 & $112.40 \pm 2.46^{c}$ & $135.80 \pm 4.87^{\mathrm{b}}$ & $118.60 \pm 4.01^{\mathrm{c}}$ & $156.20 \pm 3.22^{\mathrm{a}}$ \\
\hline \multirow[t]{3}{*}{ Platelets $\left(\mathrm{n} \times \mathbf{1 0}^{\mathbf{3}} / \mathrm{ml}\right)$} & $2 w$ & $123.80 \pm 0.80^{c}$ & $154.60 \pm 21.20^{\mathrm{a}}$ & $133.20 \pm 4.11^{\mathrm{b}}$ & $159.20 \pm 10.59^{a}$ \\
\hline & $4 w$ & $140.20 \pm 7.97^{b}$ & $279.40 \pm 33.34^{\mathrm{a}}$ & $146.60 \pm 4.17^{b}$ & $257.40 \pm 18.54^{\mathrm{a}}$ \\
\hline & $6 w$ & $136.40 \pm 4.91^{b}$ & $223.20 \pm 14.82^{\mathrm{a}}$ & $144.60 \pm 7.35^{b}$ & $228.80 \pm 31.93^{\mathrm{a}}$ \\
\hline
\end{tabular}

The different litters in the same column means significance at $(\mathrm{p}<0.05)$

$($ Mean \pm S.E) $\quad n=5$ 
Table (3): The effect of oral administration of Pediococcus $\left(23 \times 10^{6} \mathrm{CFU} /\right.$ animal) in drinking milk once daily for one month on total and differential leucocytic counts in cow calves.

\begin{tabular}{|c|c|c|c|c|c|}
\hline Items & Time (Week) & Control male & Treated male & Control female & Treated female \\
\hline \multirow{4}{*}{$\begin{array}{l}\text { WBCs } \\
\left(\mathbf{n} \times \mathbf{1 0}^{3} / \mathrm{ml}\right)\end{array}$} & 0 & $5.26 \pm 0.32^{b}$ & $7.19 \pm 0.53^{\mathrm{ab}}$ & $6.00 \pm 0.41^{b}$ & $8.67 \pm 1.14^{\mathrm{a}}$ \\
\hline & $2^{\text {nd }} w$ & $5.20 \pm 0.03^{b}$ & $6.40 \pm 0.30^{b}$ & $5.46 \pm 0.32^{b}$ & $9.98 \pm 1.27^{\mathrm{a}}$ \\
\hline & $4^{\text {th }} w$ & $5.22 \pm 0.32^{c}$ & $7.25 \pm 0.39^{b}$ & $5.70 \pm 0.37^{c}$ & $8.80 \pm 0.62^{a}$ \\
\hline & $6^{\text {th }} w$ & $6.12 \pm 0.40^{\mathrm{ab}}$ & $7.24 \pm 1.02^{\mathrm{a}}$ & $4.40 \pm 0.43^{b}$ & $7.68 \pm 0.78^{\mathrm{a}}$ \\
\hline \multirow[t]{4}{*}{ Neutrophil (\%) } & 0 & $51.40 \pm 2.16^{b}$ & $57.00 \pm 2.47^{\mathrm{a}}$ & $55.60 \pm 0.51^{\mathrm{ab}}$ & $61.00 \pm 2.66^{\mathrm{a}}$ \\
\hline & $2^{\text {nd }} w$ & $54.40 \pm 1.16^{a}$ & $55.00 \pm 3.58^{\mathrm{a}}$ & $52.20 \pm 0.37^{a}$ & $50.40 \pm 2.77^{\mathrm{a}}$ \\
\hline & $4^{\text {th }} w$ & $54.60 \pm 0.86^{\mathrm{a}}$ & $50.60 \pm 4.41^{\mathrm{a}}$ & $58.40 \pm 1.36^{\mathrm{a}}$ & $52.60 \pm 2.08^{\mathrm{a}}$ \\
\hline & $6^{\text {th }} w$ & $56.00 \pm 1.48^{a}$ & $53.20 \pm 2.70^{\mathrm{ab}}$ & $49.60 \pm 1.50^{b}$ & $52.60 \pm 2.86^{\mathrm{ab}}$ \\
\hline \multirow[t]{4}{*}{ Lymphocyte (\%) } & 0 & $36.40 \pm 1.03^{\mathrm{a}}$ & $34.80 \pm 3.48^{\mathrm{ab}}$ & $39.40 \pm 1.76^{\mathrm{a}}$ & $31.20 \pm 2.63^{b}$ \\
\hline & $2^{\text {nd }} w$ & $40.80 \pm 1.49^{a}$ & $40.20 \pm 2.87^{\mathrm{a}}$ & $43.80 \pm 1.68^{\mathrm{a}}$ & $44.40 \pm 2.50^{\mathrm{a}}$ \\
\hline & $4^{\text {th }} w$ & $40.40 \pm 0.93^{\mathrm{ab}}$ & $42.40 \pm 3.97^{\mathrm{a}}$ & $37.00 \pm 0.93^{b}$ & $42.40 \pm 2.77^{\mathrm{a}}$ \\
\hline & $6^{\text {th }} w$ & $39.60 \pm 0.68^{\mathrm{a}}$ & $40.80 \pm 2.96^{\mathrm{a}}$ & $44.00 \pm 1.08^{\mathrm{a}}$ & $41.00 \pm 2.55^{\mathrm{a}}$ \\
\hline \multirow[t]{4}{*}{ Monocyte (\%) } & 0 & $7.80 \pm 0.66^{a}$ & $5.80 \pm 0.58^{a}$ & $4.20 \pm 0.86^{\mathrm{a}}$ & $5.00 \pm 1.00^{\mathrm{a}}$ \\
\hline & $2^{\text {nd }} w$ & $3.60 \pm 0.50^{\mathrm{a}}$ & $3.40 \pm 0.60^{\mathrm{a}}$ & $3.00 \pm 0.89^{\mathrm{a}}$ & $4.00 \pm 0.70^{\mathrm{a}}$ \\
\hline & $4^{\text {th }} w$ & $3.80 \pm 0.66^{\mathrm{a}}$ & $4.60 \pm 0.50^{\mathrm{a}}$ & $3.60 \pm 0.40^{\mathrm{a}}$ & $3.60 \pm 0.50^{a}$ \\
\hline & $6^{\text {th }} w$ & $3.80 \pm 0.37^{\mathrm{a}}$ & $4.00 \pm 0.31^{\mathrm{a}}$ & $5.80 \pm 0.37^{\mathrm{a}}$ & $4.00 \pm 0.70^{\mathrm{a}}$ \\
\hline \multirow[t]{4}{*}{ Eosinophil (\%) } & 0 & $2.00 \pm 0.00^{\mathrm{ab}}$ & $1.20 \pm 0.48^{\mathrm{ab}}$ & $0.40 \pm 0.40^{b}$ & $1.60 \pm 0.40^{a}$ \\
\hline & $2 w$ & $0.80 \pm 0.20^{\mathrm{a}}$ & $1.00 \pm 0.31^{\mathrm{a}}$ & $0.40 \pm 0.24^{\mathrm{a}}$ & $0.60 \pm 0.24^{a}$ \\
\hline & $4 w$ & $0.80 \pm 0.37^{\mathrm{ab}}$ & $1.40 \pm 0.24^{\mathrm{a}}$ & $0.40 \pm 0.24^{b}$ & $0.80 \pm 0.37^{\mathrm{ab}}$ \\
\hline & $6 w$ & $0.60 \pm 0.40^{b}$ & $1.20 \pm 0.20^{\mathrm{ab}}$ & $0.40 \pm 0.40^{b}$ & $2.00 \pm 0.44^{a}$ \\
\hline \multirow[t]{4}{*}{ Basophil (\%) } & 0 & $2.40 \pm 1.91^{\mathrm{a}}$ & $1.20 \pm 0.20^{b}$ & $0.40 \pm 0.24^{c}$ & $1.20 \pm 0.58^{b}$ \\
\hline & $2 w$ & $0.40 \pm 0.24^{\mathrm{a}}$ & $0.40 \pm 0.24^{\mathrm{a}}$ & $0.60 \pm 0.24^{\mathrm{a}}$ & $0.60 \pm 0.40^{\mathrm{a}}$ \\
\hline & $4 w$ & $0.40 \pm 0.24^{\mathrm{a}}$ & $1.00 \pm 0.31^{\mathrm{a}}$ & $0.60 \pm 0.40^{\mathrm{a}}$ & $0.60 \pm 0.24^{\mathrm{a}}$ \\
\hline & $6 w$ & $0.00 \pm 0.00^{b}$ & $0.80 \pm 0.37^{\mathrm{a}}$ & $0.20 \pm 0.37^{\mathrm{ab}}$ & $0.40 \pm 0.24^{\mathrm{ab}}$ \\
\hline
\end{tabular}

The different litters in the same column means significance at $(\mathrm{p}<0.05)$

(Mean \pm S.E) $\quad n=5$

Table (4): The effect of oral administration of Pediococcus $\left(23 \times 10^{6} \mathrm{CFU} /\right.$ animal $)$ in drinking milk once daily for one month on creatinine and urea in cow calves.

\begin{tabular}{|c|c|c|c|c|c|}
\hline Items & Time (Week) & Control male & Treated male & Control female & Treated female \\
\hline \multirow{4}{*}{$\begin{array}{l}\text { CR-S } \\
(\mathrm{mg} / \mathrm{dl})\end{array}$} & 0 & $1.08 \pm 0.05^{\mathrm{a}}$ & $1.20 \pm 0.04^{\mathrm{a}}$ & $1.10 \pm 0.07^{\mathrm{a}}$ & $1.14 \pm 0.04^{\mathrm{a}}$ \\
\hline & $2^{\text {nd }} w$ & $1.12 \pm 0.05^{\mathrm{a}}$ & $1.22 \pm 0.02^{\mathrm{a}}$ & $1.10 \pm 0.06^{\mathrm{a}}$ & $1.22 \pm 0.02^{\mathrm{a}}$ \\
\hline & $4^{\text {th }} w$ & $1.00 \pm 0.13^{\mathrm{a}}$ & $1.06 \pm 0.02^{\mathrm{a}}$ & $0.72 \pm 0.11^{b}$ & $1.08 \pm 0.03^{\mathrm{a}}$ \\
\hline & $6^{\text {th }} w$ & $0.88 \pm 0.05^{a}$ & $0.94 \pm 0.02^{\mathrm{a}}$ & $0.90 \pm 0.04^{\mathrm{a}}$ & $1.02 \pm 0.06^{\mathrm{a}}$ \\
\hline \multirow{4}{*}{$\begin{array}{l}\text { Urea } \\
\text { ( } \mathrm{mg} / \mathrm{dl})\end{array}$} & 0 & $9.80 \pm 0.48^{b}$ & $13.40 \pm 0.81^{\mathrm{a}}$ & $9.60 \pm 0.81^{b}$ & $13.00 \pm 0.70^{\mathrm{a}}$ \\
\hline & $2^{\text {nd }} w$ & $10.60 \pm 0.40^{b}$ & $14.20 \pm 0.37^{\mathrm{a}}$ & $10.40 \pm 0.40^{b}$ & $15.40 \pm 0.60^{\mathrm{a}}$ \\
\hline & $4^{\text {th }} w$ & $14.40 \pm 0.92^{\mathrm{a}}$ & $9.00 \pm 0.44^{b}$ & $16.00 \pm 0.70^{\mathrm{a}}$ & $10.80 \pm 0.80^{b}$ \\
\hline & $6^{\text {th }} w$ & $18.60 \pm 0.60^{\mathrm{a}}$ & $11.40 \pm 0.50^{b}$ & $17.60 \pm 0.67^{\mathrm{a}}$ & $12.80 \pm 0.58^{b}$ \\
\hline
\end{tabular}

The different litters in the same column means significance at $(\mathrm{p}<0.05)$

(Mean \pm S.E) $\quad \mathrm{n}=5$ 
Table (5): The effect of oral administration of Pediococcus $\left(23 \times 10^{6} \mathrm{CFU} /\right.$ animal $)$ in drinking milk once daily for one month on total protein and their fractions in cow calves.

\begin{tabular}{|c|c|c|c|c|c|}
\hline Items & Time (Week) & Control male & Treated male & Control female & Treated female \\
\hline \multirow{4}{*}{$\mathrm{TP}(\mathrm{g} / \mathrm{dl})$} & 0 & $4.58 \pm 0.05^{\mathrm{a}}$ & $4.78 \pm 0.12^{\mathrm{a}}$ & $4.62 \pm 0.18^{\mathrm{a}}$ & $4.96 \pm 0.18^{\mathrm{a}}$ \\
\hline & $2^{\text {nd }} w$ & $4.72 \pm 0.15^{b}$ & $5.30 \pm 0.14^{\mathrm{a}}$ & $4.98 \pm 0.04^{\mathrm{ab}}$ & $5.34 \pm 0.24^{\mathrm{a}}$ \\
\hline & $4^{\text {th }} w$ & $4.86 \pm 0.10^{b}$ & $7.84 \pm 0.24^{\mathrm{a}}$ & $4.58 \pm 0.21^{b}$ & $7.98 \pm 0.08^{a}$ \\
\hline & $6^{\text {th }} w$ & $4.94 \pm 0.18^{b}$ & $8.50 \pm 0.19^{\mathrm{a}}$ & $4.78 \pm 0.07^{b}$ & $8.08 \pm 0.04^{a}$ \\
\hline \multirow{5}{*}{$\begin{array}{l}\text { ALB } \\
(\mathrm{g} / \mathrm{dl})\end{array}$} & 0 & $2.60 \pm 0.15^{b}$ & $2.80 \pm 0.05^{\mathrm{ab}}$ & $2.88 \pm 0.06^{\mathrm{a}}$ & $2.90 \pm 0.06^{\mathrm{a}}$ \\
\hline & $2^{\text {nd }} w$ & $2.94 \pm 0.05^{b}$ & $3.22 \pm 0.08^{\mathrm{a}}$ & $2.90 \pm 0.06^{b}$ & $3.18 \pm 0.06^{\mathrm{a}}$ \\
\hline & $4^{\text {th }} w$ & $2.96 \pm 0.06^{b}$ & $4.60 \pm 0.07^{\mathrm{a}}$ & $3.02 \pm 0.07^{b}$ & $4.84 \pm 0.08^{\mathrm{a}}$ \\
\hline & $6^{\text {th }} w$ & $2.96 \pm 0.06^{b}$ & $4.88 \pm 0.12^{\mathrm{a}}$ & $2.82 \pm 0.05^{b}$ & $4.76 \pm 0.10^{a}$ \\
\hline & 0 & $1.98 \pm 0.15^{\mathrm{a}}$ & $1.98 \pm 0.12^{\mathrm{a}}$ & $1.74 \pm 0.22^{a}$ & $2.06 \pm 0.20^{a}$ \\
\hline \multirow{3}{*}{$\begin{array}{l}\text { Globulin } \\
\text { (g/dl) }\end{array}$} & $2^{\text {nd }} w$ & $1.78 \pm 0.15^{\mathrm{a}}$ & $2.08 \pm 0.12^{\mathrm{a}}$ & $2.08 \pm 0.06^{a}$ & $2.16 \pm 0.26^{a}$ \\
\hline & $4^{\text {th }} w$ & $1.9 \pm 0.12^{b}$ & $3.25 \pm 0.20^{\mathrm{a}}$ & $1.56 \pm 0.16^{b}$ & $3.14 \pm 0.14^{a}$ \\
\hline & $6^{\text {th }} w$ & $1.98 \pm 0.15^{\mathrm{b}}$ & $3.62 \pm 0.12^{\mathrm{a}}$ & $1.96 \pm 0.12^{b}$ & $3.32 \pm 0.07^{\mathrm{a}}$ \\
\hline
\end{tabular}

The different litters in the same column means significance at $(p<0.05)$

(Mean \pm S.E) $\quad n=5$

Table (6): The effect of oral administration of Pediococcus $\left(23 \times 10^{6}\right.$ CFU/animal) in drinking milk once daily for one month on some liver functions in cow calves.

\begin{tabular}{|c|c|c|c|c|c|}
\hline Items & $\begin{array}{l}\text { Time } \\
\text { (Week) }\end{array}$ & Control male & Treated male & Control female & Treated female \\
\hline \multirow{5}{*}{$\begin{array}{l}\text { ALT } \\
\text { (IU/L) }\end{array}$} & 0 & $25 \pm 4.33^{\mathrm{a}}$ & $25.6 \pm 3.94^{\mathrm{a}}$ & $20.2 \pm 2.13^{\mathrm{a}}$ & $24.4 \pm 3.11^{\mathrm{a}}$ \\
\hline & $2^{\text {nd }} w$ & $18.4 \pm 1.32^{\mathrm{a}}$ & $20 \pm 1.30^{\mathrm{a}}$ & $17.4 \pm 1.16^{\mathrm{a}}$ & $19.2 \pm 0.58^{\mathrm{a}}$ \\
\hline & $4^{\text {th }} \mathrm{w}$ & $16 \pm 0.70^{\mathrm{b}}$ & $20 \pm 1.14^{\mathrm{a}}$ & $19.8 \pm 0.86^{\mathrm{a}}$ & $19.6 \pm 0.51^{\mathrm{a}}$ \\
\hline & $6^{\text {th }} \mathrm{w}$ & $17.4 \pm 1.02^{\mathrm{a}}$ & $18.4 \pm 1.69^{\mathrm{a}}$ & $15.2 \pm 1.59^{\mathrm{a}}$ & $18.4 \pm 1.12^{\mathrm{a}}$ \\
\hline & 0 & $29.80 \pm 0.73^{\mathrm{a}}$ & $29.00 \pm 2.16^{a}$ & $31.20 \pm 1.59^{\mathrm{a}}$ & $34.00 \pm 3.08^{\mathrm{a}}$ \\
\hline \multirow{2}{*}{$\begin{array}{l}\text { AST } \\
\text { (IU/L) }\end{array}$} & $\begin{array}{l}2^{\text {nd }} w \\
4^{\text {th }} w\end{array}$ & $\begin{array}{l}29.40 \pm 1.16^{b} \\
29.80 \pm 0.73^{a}\end{array}$ & $\begin{array}{l}38.80 \pm 3.18^{a} \\
31.20 \pm 2.05^{a}\end{array}$ & $\begin{array}{l}29.20 \pm 0.58^{b} \\
24.40 \pm 1.53^{b}\end{array}$ & $\begin{array}{l}40.20 \pm 1.46^{\mathrm{a}} \\
24.60 \pm 1.02^{\mathrm{b}}\end{array}$ \\
\hline & $6^{\text {th }} \mathrm{W}$ & $24.20 \pm 0.37^{\mathrm{a}}$ & $24.40 \pm 1.72^{\mathrm{a}}$ & $21.80 \pm 0.80^{a}$ & $21.20 \pm 1.24^{\mathrm{a}}$ \\
\hline \multirow{3}{*}{ ALP(IU/L) } & $\begin{array}{l}0 \\
2^{\text {nd }} w\end{array}$ & $\begin{array}{l}591.80 \pm 62.74^{a b} \\
531.80 \pm 23.60^{b}\end{array}$ & $\begin{array}{l}591.00 \pm 76.87^{\mathrm{ab}} \\
461.80 \pm 42.90^{b}\end{array}$ & $\begin{array}{l}344.80 \pm 19.13^{b} \\
438.00 \pm 4.31^{b}\end{array}$ & $\begin{array}{l}868.60 \pm 171.33^{a} \\
776.00 \pm 79.59^{a}\end{array}$ \\
\hline & $4^{\text {th }} \mathrm{w}$ & $327.20 \pm 46.91^{b c}$ & $473.20 \pm 87.38^{\mathrm{ab}}$ & $264.80 \pm 37.09^{c}$ & $631.00 \pm 33.51^{a}$ \\
\hline & $6^{\text {th }} \mathrm{w}$ & $223.80 \pm 20.74^{b}$ & $300.60 \pm 46.59^{a b}$ & $222.00 \pm 4.41^{b}$ & $388.20 \pm 95.30^{\mathrm{a}}$ \\
\hline
\end{tabular}

The different litters in the same column means significance at $(p<0.05)$

$($ Mean \pm S.E) $\quad n=5$ 
Table (7): The effect of oral administration of Pediococcus $\left(23 \times 10^{6} \mathrm{CFU} / \mathrm{animal}\right)$ in drinking milk once daily for one month on blood electrolytes in cow calves.

\begin{tabular}{|c|c|c|c|c|c|}
\hline Items & Time (Week) & Control male & Treated male & Control female & Treated female \\
\hline \multirow{4}{*}{ Sodium( $\mathrm{mEq} / \mathrm{L}$ ) } & 0 & $97.8 \pm 7.33^{\mathrm{a}}$ & $105.2 \pm 8.88^{a}$ & $86.6 \pm 6.01^{a}$ & $103.4 \pm 9.71^{\mathrm{a}}$ \\
\hline & $2^{\text {nd }} w$ & $96 \pm 2.98^{a}$ & $107.8 \pm 10.22^{\mathrm{a}}$ & $92.2 \pm 2.56^{a}$ & $108.6 \pm 11.07^{a}$ \\
\hline & $4^{\text {th }} w$ & $77.6 \pm 1.96^{b}$ & $85.6 \pm 4.19^{a}$ & $70.6 \pm 0.40^{b}$ & $89.8 \pm 2.51^{a}$ \\
\hline & $6^{\text {th }} \mathrm{w}$ & $87.2 \pm 1.02^{b}$ & $106.2 \pm 1.77^{\mathrm{a}}$ & $94 \pm 2.47^{b}$ & $105.6 \pm 3.96^{\mathrm{a}}$ \\
\hline \multirow{4}{*}{ Potassium(mEq/L) } & 0 & $4 \pm 0.20^{b}$ & $4.42 \pm 0.15^{\mathrm{ab}}$ & $4.88 \pm 0.22^{a}$ & $4.96 \pm 0.37^{a}$ \\
\hline & $2^{\text {nd }} w$ & $5.56 \pm 0.17^{b}$ & $6.34 \pm 0.22^{a}$ & $5.06 \pm 0.22^{b}$ & $6.2 \pm 0.37^{a}$ \\
\hline & $4^{\text {th }} \mathrm{w}$ & $4.7 \pm 0.23^{a}$ & $3.47 \pm 0.02^{b}$ & $4.92 \pm 0.17^{a}$ & $3.22 \pm 0.15^{b}$ \\
\hline & $6^{\text {th }} \mathrm{w}$ & $4.724 \pm 0.20^{a}$ & $3.08 \pm 0.11^{b}$ & $4.58 \pm 0.16^{a}$ & $2.98 \pm 0.06^{b}$ \\
\hline \multirow{4}{*}{ Calcium( mg/L) } & 0 & $10.18 \pm 0.18^{a}$ & $9.78 \pm 0.16^{\mathrm{a}}$ & $10.32 \pm 0.27^{a}$ & $9.7 \pm 0.27^{\mathrm{a}}$ \\
\hline & $2^{\text {nd }} w$ & $9.62 \pm 0.20^{\mathrm{ab}}$ & $9.94 \pm 0.28^{a}$ & $9.34 \pm 0.06^{b}$ & $10.04 \pm 0.12^{a}$ \\
\hline & $4^{\text {th }} \mathrm{w}$ & $8.7 \pm 0.20^{b}$ & $9.61 \pm 0.27^{a}$ & $9.24 \pm 0.19^{\mathrm{ab}}$ & $9.46 \pm 0.08^{a}$ \\
\hline & $6^{\text {th }} \mathrm{w}$ & $8.86 \pm 0.30^{b}$ & $9.64 \pm 0.27^{\mathrm{a}}$ & $8.5 \pm 0.11^{b}$ & $9.4 \pm 0.28^{\mathrm{a}}$ \\
\hline \multirow{4}{*}{$\begin{array}{l}\text { Phosphorus } \\
\text { ( } \mathrm{mg} / \mathrm{L})\end{array}$} & 0 & $7.84 \pm 0.20^{b}$ & $8.42 \pm 0.25^{\mathrm{ab}}$ & $8.44 \pm 0.22^{a b}$ & $8.92 \pm 0.37^{a}$ \\
\hline & $2^{\text {nd }} w$ & $8.56 \pm 0.33^{a}$ & $8.94 \pm 0.30^{a}$ & $8.58 \pm 0.16^{a}$ & $9.42 \pm 0.40^{a}$ \\
\hline & $4^{\text {th }} w$ & $8.26 \pm 0.19^{b}$ & $9.09 \pm 0.13^{a}$ & $7.98 \pm 0.14^{b}$ & $8.84 \pm 0.17^{a}$ \\
\hline & $6^{\text {th }} w$ & $7.9 \pm 0.19^{b}$ & $9.1 \pm 0.27^{\mathrm{a}}$ & $7.98 \pm 0.37^{b}$ & $9.6 \pm 0.39^{a}$ \\
\hline
\end{tabular}

The different litters in the same column means significance at $(p<0.05)$

(Mean \pm S.E) $\quad n=5$

Table (8): The effect of oral administration of Pediococcus $\left(23 \times 10^{6} \mathrm{CFU} /\right.$ animal) in drinking milk once daily for one month on bodyweight in cow calves.

\begin{tabular}{|c|c|c|c|c|c|}
\hline Items & Time (Week) & Control male & Treated male & Control female & Treated female \\
\hline \multirow{8}{*}{ Body weight(kg) } & 0 & $83.2 \pm 1.39^{\mathrm{a}}$ & $76.6 \pm 5.98^{\mathrm{a}}$ & $79.6 \pm 2.18^{a}$ & $76.2 \pm 6.37^{\mathrm{a}}$ \\
\hline & $2^{\text {nd }} w$ & $82.2 \pm 2.83^{\mathrm{a}}$ & $92.4 \pm 8.70^{a}$ & $87 \pm .836^{a}$ & $92.6 \pm 4.61^{\mathrm{a}}$ \\
\hline & $4^{\text {th }} w$ & $100.2 \pm 3.28^{b}$ & $122 \pm 8.45^{\mathrm{a}}$ & $100 \pm 1.70^{b}$ & $116 \pm 5.099^{\mathrm{a}}$ \\
\hline & $6^{\text {th }} w$ & $105 \pm 1.61^{b}$ & $134.2 \pm 9.91^{\mathrm{a}}$ & $103.8 \pm 2.00^{\mathrm{b}}$ & \multirow{2}{*}{$\begin{array}{l}133.6 \pm 6.70^{\mathrm{a}} \\
180 \pm 6.71^{\mathrm{ab}}\end{array}$} \\
\hline & $14^{\text {th }} w$ & $175 \pm 2.98^{b}$ & $196 \pm 9.66^{\mathrm{a}}$ & $163.6 \pm 2.69^{b}$ & \\
\hline & $22^{\text {th }} w$ & $271 \pm 2.68^{b}$ & $329 \pm 3.31^{a}$ & $224.8 \pm 5.54^{c}$ & $260.6 \pm 3.85^{\mathrm{b}}$ \\
\hline & $37^{\text {th }} w$ & $359.6 \pm 1.75^{c}$ & $419.6 \pm 9.17^{\mathrm{a}}$ & $333.8 \pm 6.64^{d}$ & $387 \pm 2.53^{b}$ \\
\hline & $47^{\text {th }} w$ & $402.4 \pm 4.58^{b}$ & $477.6 \pm 8.35^{\mathrm{a}}$ & $359.4 \pm 2.54^{c}$ & $399 \pm 9.00^{b}$ \\
\hline
\end{tabular}

The different litters in the same column means significance at $(p<0.05)$

(Mean \pm S.E) $\quad n=5$

\section{DISCUSSION}

Our results showed a significant increase in total erythrocytes count in treated groups compared to control groups at zero and fourth weeks, while a significant decrease in treated groups compared to control groups was recorded at second week. Also data reflected a significant increase in treated female group compared to control groups and a significant increase in treated male group compared to control male group at sixth week.

But there is a significant increase in hemoglobin content in treated female group compared to control groups at zero week, also showed a significant increase in treated male group compared to control female group. While a non-significant 
changes was recorded at second and sixth week's.Moreover, showed a significant increase in control male group compared to control female group at fourth week.

The significant increase in packed cell volume at zero and fourth weeks in treated female group compared to control male group .While a non-significant changes was recorded at second week, but recorded a significant increase in treated female group compared to control female group at sixth week. Table (1)

Our data was in agreement with Salahuddin et al., (2013) and Shareef and Dabbagh (2009) who concluded that, the feeding of commercial ration with different level of probiotic in mice improved Total erythrocyte count and hemoglobin concentration. Probiotics supplementation helps in improving normal hematology.

Moreover Doaa and Moshira (2015) stated that, rabbits received mixture of pre and probiotic recorded the highest value of RBCS count, PCV, $\mathrm{Hb}$ concentration.

But our data disagree with Sarah et al., (2017) reported that lactating Holstein-Friesian cows received oral probiotic administration (containing Lactobacillus acidophilus, Saccharomyces cerevisiae, Enterococcus faecium, Aspergillus oryza and Bacillus subtilis) had no effect on PCV.

Looking to Table( 2) there is a significant increase in mean corpuscular volume in treated groups compared to control groups at zero, second, fourth and sixth weeks.

Result evaluated a significant increase in mean corpuscular hemoglobin in treated groups compared to control groups at zero, second, fourth and sixth week, but at zero week only showed a significant decrease in control female group compared to control male group .

Moreover there is a significant increase in mean corpuscular hemoglobin concentration in treated male group compared to control groups at zero week, but showed a significant decrease in control male group compared to treated groups. Data recorded a significant increase in treated groups compared to control groups at second, fourth and sixth weeks, but at fourth week only showed a significant decrease in control male group compared to other groups.

The present data reflected a significant increase in thrombocyte count at zero, second, fourth and sixth week in treated groups compared to the control groups, but at zero week only showed a significant increase in treated male group compared to control groups and at second week only showed a significant increase in control female group compared to control male group.

Table( 3)showed a significant increase in total leucocytes counts in treated female group compared to control groups at zero week. While showed a significant increase at second and fourth week in treated female group compared to the others groups but recorded at fourth group only a significant increase in treated male group compared to control groups. Data showed a significant decrease in control female group compared to other groups at sixth week.

Same data also reported that Doaa and Moshira (2015) who stated that, rabbits received mixture of pre and probiotic recorded the highest value of Total Leucocyte Count. Moreover Al-Saad et al.,(2014) also showed a significant increase in number of white Blood Cells(WBC) in blood samples of probiotic and organic acids groups compared to antibiotic group, It seems that the microbial interactions and effects on local immune stimulation results increase of white blood cells and immunity these results are accordance with Endings of Zareshahnehet al.,(2007) and EFSA(2010-b) but are not accordance with the findings of Shareef and ADabbagh(2009).

Our results detected a significant increase in neutrophil percent in treated groups compared to the control male group at zero week, while at second and fourth week showed a nonsignificant changes in all groups. Data evaluated a significant increase in control male group compared to control female group at sixth week. While lymphocyte showed a significant increase in control groups compared to treated female groups at zero week but a non-significant changes in lymphocyte percent at second and sixth weeks in all groups, while in other side there are a significant increase in treated groups compared to control female group at fourth week.

These data agree with Doaa and Moshira (2015) stated a high value in heterophils and lymphocytes number in rabbits received mixture of pre and probiotic. Same data obtained by Sarah et al., (2017) who evaluated that oral probiotic administration (containing Lactobacillus acidophilus, Saccharomyces cerevisiae, Enterococcus faecium, Aspergillus oryza and Bacillus subtilis) on lactating Holstein-Friesian cows had a significant increase in per cent lymphocyte count, and a significant decrease in per cent neutrophil count in probiotictreated animals.

Our results detected a non-significant changes in monocyte percent at zero, second, fourth and sixth weeks in all groups. Our data mirrored a significant increase in eosinophil percent at zero week in treated female group compared to control female group, and detected a nonsignificant change in all groups at second week, while a significant increase was recorded in treated male group compared to control female group at fourth week. But at sixth week detected a significant increase in treated female group compared to control groups.

Result evaluated a significant increase in basophil percent in control male group compared to other groups and a significant increase in treated groups compared to control female groups at zero week and a non-significant changes at second and fourth week in all groups, but showed a significant 
increase in treated male group compared to control male group at sixth week.

In Table (4) there were a non-significant changes in creatinine at zero, second and sixth weeks in all groups, but a significant decrease was recorded in control female group compared to the others groups at fourth week. The results showed a significant increase in urea at zero and second weeks in treated groups compared to control groups, but showed a significant decrease in treated groups compared to control groups at fourth and sixth weeks.

Our data was in agreement with Kehoe et al., (2008) who detected that calves supplemented with purified nucleotides had higher in BUN-to-creatinine ratio and decreased in kidney functions. Not only Patterson and Burkholder (2003) recorded lower serum cholesterol Decrease ammonia and urea excretion and lower skatol, indole and phenol in cattle supplemented with probiotics, but also Doaa and Moshira (2015) who stated that, rabbits received mixture of pre and probiotic recorded a significant decrease in total cholesterol, creatinine and urea. Dietary supplementation of prebiotic and probiotic and their mixture improves kidney functions. However Saleem et al., (2017) concluded that, lambs that received PRO (a commercial probiotic containing a mixture of two strains of Pediococcus, Pediococcus acidilactici and Pediococcus Pentosaceus, with dextrose as the carrier compound) in post-weaning diet appeared to show a significant decrease in blood urea and cholesterol concentration.

In Table (5) there were non-significant changes in total protein at zero week in all groups, but showed a significant decrease in control male compared to the other groups at second week. Moreover a significant increase was detected in treated groups compared to control groups at fourth and sixth week.

In the same ground our results were supported by the results of Ezema (2014) who recorded that, Probiotic supplementation in broiler chicken significantly increased total proteins. But Sarah et al., (2017) reported that oral probiotic administration (containing Lactobacillus acidophilus, Saccharomyces cerevisiae, Enterococcus faecium, Aspergillus oryza and Bacillus subtilis) on lactating Holstein-Friesian cows had no effect on total protein concentration in plasma.

Our results showed a significant decrease in albumin in control male group compared to control and treated female groups at zero week, but at second, fourth and sixth weeks showed a significant increase in treated groups compared to control groups. There were a non-significant changes in globulin in all groups at zero and second weeks, but detected a significant increase in treated groups compared to control groups at fourth and sixth weeks.

Our results showed non-significant changes in serum alanine transaminase ALT in all groups at zero, second and sixth weeks while data showed a significant decrease in control male group compared to the others groups at fourth week, Table (6). The present work reflected that nonsignificant changes at zero and sixth weeks in serum aspartate transaminase (AST) in all groups, while a significant increase was evaluated at second week in treated groups compared to control groups.

Result also detected a significant increase in control and treated male groups compared to control and treated female groups, also was recorded a non-significant changes between treated male group and control male group, and between treated female group and control female group at fourth week.

Our data detected a significant increase in serum alkaline phosphatase (ALP) in treated female group compared to control female group at zero week, while at second week showed a significant increase in treated female group compared to other groups. Data showed a significant increase in treated female group compared to control groups at fourth and sixth week, but showed only at fourth week a significant decrease in control female group compared to treated groups. (Table (6)

Similar findings were reported with Xing et al., (2006).who stated that consumption of Bifidobacterium Catenulate and Lactobacillus Fermentum improves liver functions. Also the use of Lactobacillus plantarum and Bifidobacterium infantis in rats induced liver injury improved. Osman et al., (2007). Not only administration of heat-killed Lactobacillus brevis (dosage of 100 or $500 \mathrm{mg} / \mathrm{kg}$ once a day) after 35 days was inhibited an increase in serum ALT and AST levels in alcoholic liver disease using ethanol-containing diet-fed mice Segawa et al., (2008), but also there were an inhibition of TNF- $\alpha$ and sterol regulatory element-binding protein (SREBPs) up-regulation by Lactobacillus brevis, which in turn may decrease serum ALT levels. Furthermore, elevated ALT, a sign of hepatocyte damage results from damaged biological membranes. Larson et al., (2008)

The decrease in serum ALT levels was seen with Bifidobacterium pseudocatenulatum, Bifidobacterium longum and Bifidobacterium longum $\left(10^{8}-10^{9} \mathrm{CFU}\right)$ after 7 weeks in high fat diet-induced obese rats. An HM et al., (2011). These data agree with the results of Doaa and Moshira (2015) found a significant decrease in alanine amino transferase (ALT), aspartate amino transferase (AST) and improves in cell-mediated immune response and liver function in rabbits received mixture of pre and probiotic.

In Table (7) results showed non-significant changes in sodium in all groups at zero and second weeks, while at fourth and sixth weeks showed a significant increase in treated groups compared to control groups. 
Our results match with the results of Schroeder et al., (2004) who concluded that. Boulardii has specific durationdependent effects on the secretory response of the pig jejunal mucosa which developed during 8-day treatment and significant increase $\mathrm{Na}+$ and $\mathrm{Cl}$ - but disappeared during further application. Thus, this study supports the concept that probiotics may exert beneficial effects in the gastrointestinal tract. Similar findings were seen with consumption of strain Enterococcus faecium EK13 (dosage $10^{9} \mathrm{CFU} / \mathrm{ml}$ ) in piglets for 14 days Strompfova et al., (2006).

In Table (7) also we found a significant decrease in Potassium in control male group compared to control and treated female groups at zero week, while a significant increase was recorded in treated groups compared to control groups at second week, but detected a significant increase in control groups compared to treated groups at fourth and sixth weeks. Our results showed a non-significant changes in Calcium in all groups at zero week, while detected a significant increase in treated groups compared to control female group at second week. A significant increase was recorded in treated groups compared to control male group at fourth week, but at sixth week reflected a significant increase in treated groups compared to control groups. However Phosphorus showed a significant increase in treated female group compared to control male group at zero week, while at second week showed a non-significant changes in all group, moreover a significant increase was detected in treated groups compared to control groups at fourth and sixth weeks.

Our results in agreement with Patterson and Burkholder (2003) who detected that, probiotics and prebiotics improve mineral absorption. Also Ateequr et al.,(2012) who showed that administration of the probiotic mixture (containing the 8 bacterial strains Streptococcus thermophilus, Bifidobacterium breve, Bifidobacterium longum , Bifidobacterium infantis

Lactobacillus acidophilus, Lactobacillus plantarum , Lactobacillus paracasei and Lactobacillus delbrueckii sub sp. Bulgaricus) parallel with the clindamycin therapy had a beneficial and stabilizing effect on the intestinal metabolic homeostasis by decreasing toxic metabolites and protecting the endogenic microbiota from destruction. Probiotics could be a reasonable strategy in prevention of antibiotic associated disturbances of the intestinal homeostasis and disorders.

Our results showed non-significant changes in body weight in all groups at zero and second week, while showed a significant increase in treated groups compared to control groups at fourth and sixth weeks. But at $14^{\text {th }}$ week there is a significant increase in treated male group compared to control groups in Table (8). There is a significant increase in treated male group compared to other groups and a significant decrease in control female group compared to other groups at $22^{\text {th }}$ and $47^{\text {th }}$ week, while showed a significant increase in treated male group compared to other groups and a significant increase in treated female group compared to other control groups at $37^{\text {th }}$ week.

Our results in agreement with Frizzo et al., (2011) who said that, lactic acid bacteria (LAB) supplementation increased body weight gain (BWG) and improve feed efficiency in calves. And confirmed with Hiruta (2015) indicated that, the usage of probiotics in ruminants stimulate forage intake, and the increase in forage intake can result in improved live weight gain. Moreover application of probiotics in receiving cattle, increase in daily gain, increase in feed consumption, and improvement in feed gain and steers fed a probiotics had greater final weight, ADG, DM intake, hot carcass weight, carcass-adjusted ADG and improved feed efficiency.

Also our results confirmed with Bhatt et al., (2016) concluded that, probiotics supplementation, more specifically Lactobacillus acidophilus (at $10^{7} \mathrm{CFU} / \mathrm{g}$ concentrate) in growing Chinchilla rabbits improved digestibility and utilization of nutrients, BW gain and feed conversion ratio. Not only agree with Ezema (2014) who said that, Probiotic supplementation in broiler chicken significantly increased weight gain ,but also agree with Corcionivoschi et al., (2010) reported that, probiotics increased food intake, and body weight. Manipulating ruminal fermentation and ruminant productivity.

\section{REFERENCES}

Al-Saad.S, M. Abbod and A. Abo Yones (2014): Effects of some Growth Promoters on Blood Hematology and Serum Composition of Broiler Chickens. International Journal of Agricultural Research9 (5):265270.ISSN1816-4897 DOI:108928/iar.261270.Ö. Acadernic Journals Inc.

An HM, Park SY, do Lee do K, Kim JR, Cha MK and Lee SW (2011): Antiobesity and lipid-lowering effects of Bifidobacterium spp. in high fat diet-induced obese rats. Lipids Health Dis. 2011; 10:116.

Ateequr Rehman, Femke Anouska Heinsen , Marjorie E. Koenen, Koen Venema, Henrik Knecht, Stephan Hellmig Stefan Schreiber and Stephan J. Ott (2012): Effects of probiotics and antibiotics on the intestinal homeostasis in a computer controlled model of the large intestine .BMC Microbiology 12:47.

Bhatt.R. S., A. R. Agrawal and A.Sahoo (2016): Effect of probiotic supplementation on growth performance, nutrient utilization and carcass characteristics of growing Chinchilla rabbits. Pages 304-309 | received 21 Mar 2015, Accepted 14 Mar 2016, Published online: 27 Apr 2016.

Corcionivoschi Nicolae, Dan Drinceanu, Ioan Mircea Pop, Deirdre Stack, Lavinia Ştef and Călin Julean Billy Bourke(2010):The Effect of probiotics on animal health .Scientific Papers: Animal Science and Biotechnologies, 43 (1)

Doaa. H. Abdelhady and Moshira A. El-Abasy (2015): Effect of Prebiotic and Probiotic on Growth, Immuno-hematological responses and Biochemical Parameters of infected rabbits with Pasteurella multocida. Benha veterinary medical journal, VOL. 28, NO. 2:40-51.

Doumas, B.T. (1975): Colorimetric determination of total proteins. Clinical. Chem, 21:1159-1166

Doumas, B.T. and Biggs, H.G. (1972): Determination of serum globulin. In Standard Method of Clinical Chemistry. Vol, 7, Edited by G.R.Cooper, New York, Academic press.

Doumas, B.T; Baysa, D.D; Carler, R.J; Peler, T. and Schaffer, R. (1981) Determination of serum albumin. Clinical Chem, 27:1642.

EFSA (European Food Safety Authority), (2010-b): Scientific Opinion on the safety and efficacy of acetic acid, sodium diacetate and calcium 
acetate as preservatives for feed for all animal species EFSA J,Vol 10.10 .2908 a efsa2012.2571.

El-Merzabani. M.M.(1977): Determination of serum phosphorus. Clin. Chem. Clin. Biochem, 15, $715-718$.

Ezema Chuka (2014): Comparative Study of the effects of probiotic and commercial enzyme on growth rate, hematology and serum biochemistry of broiler chicken. J Food Process Technol 5: 367. doi:10.4172/2157-7110.1000367.

Feldman, BF; Zinkl, JG and Jain, VC (2000): Schemes Veterinary Hematology $.5^{\text {th }}$ Ed .Lippincott Williams and Wilkins.Canda.

Fuller. R. (1989): Probiotics in man and animals .Journal of Applied Bacteriology 66, 365-378.

Frizzo.L.S,L.P.Soto,M.V.Zbrun,E.Bertozzi,G.Sequeira,R.RodríguezArmesto and M.R.Rosmini(2010):Lactic acid bacteria to improve growth performance in young calves fed milk replacer and spray-dried whey powder. Animal 157(3):159-167.

Frizzo.L.S, M.V. Zbrun, L.P. Soto, M.L. Signorin (2011): Effects of probiotics on growth performance in young calves: A meta-analysis of randomized controlled trials. November 3, Volume 169, Issues 3-4, Pages 147-156.

Gindler, M. and King, J. (1972): Chemical method for determination of calcium in serum. Am. J. Clin. Pathol, 58, 376.

Haakensen, M; Dobson, CM; Hill, JE and Ziola, B (2009). "Reclassification of Pediococcus dextrinicus (Coster and White 1964) back 1978 (Approved Lists 1980) as Lactobacillus dextrinicus comb. Nov., and emended description of the genus Lactobacillus". International Journal of Systematic and Evolutionary Microbiology. 59 (Pt 3): 615-21. Doi: 10.1099/ijs.0.65779-0. PMID 19244449.

Henry R.J. (1974): Colorimetric Determination of creatinine chemistry, principles and techniques $.2^{\text {nd }}$, Ed. Harper and Row, p.525.

Henry, R., Cannon, D.and Winkelman, J. (1974): Clinical chemistry principles and technicsHarper and Row. New York, 1547.

Hiruta Yirga (2015): The Use of probiotics in animal nutrition. J Prob Health 3:132. doi:10.4172/2329-8901.1000132.

Kehoe. S. I., A. J. Heinrichs, C. R. Baumrucker, and D. L. Greger (2008): Effects of nucleotide supplementation in milk replacer on small intestinal absorptive capacity in dairy calves.J. Dairy Sci. 91:27592770 doi:10.3168/jds.2007-0751 (C) American Dairy Science Association.

Larson-Meyer DE, Newcomer BR, Heilbronn LK, Volaufova J, Smith SR, Alfonso $\mathrm{AJ}(2008)$ : Effect of 6-month calorie restriction and exercise on serum and liver lipids and markers of liver function. Obesity (Silver Spring). 2008; 16:1355-62.

Meng. Q. W, L. Yan, X. Ao, T. X. Zhou, J. P. Wang, J. H. Lee, and I. H. Kim (2010): Influence of probiotics in different energy and nutrient density diets on growth performance, nutrient digestibility, meat quality, and blood characteristics in growing-finishing pigs.J ANIM SCl. 2010, 88:3320-3326. Doi: 10.2527/jas.2009-2308 originally published online June 18.

Osman N, Adawi D, Ahrne S, Jeppsson B and Molin G.(2007): Endotoxin- and D-galactosamine-induced liver injury improved by the administration of Lactobacillus, Bifidobacterium and blueberry. Dig Liver Dis. 2007; 39:849-56.

Patterson J. A. and Burkholder. K. M. (2003): Application of prebiotics and probiotics in poultry production. Poultry Science 82:627-631.

Patton, C. J. and Crouch, S. R. (1977): Enzymatic determination of urea. Anal. Chem.49:466-469.

Reitman, S. and Frankel, S. (1957): Colorimetric determination of glutamic oxaloacetic and glutamic pyruvic transaminases.Am.J.Clin.Path, 28;56.

Rosalki, S. (1993): Quantitative determination of alkaline phosphatase. Clin. Chem., 39/4: 648-652.

Salahuddin. M, H Akhter, S Akter, MA Miah and N Ahmad (2013): Effects of probiotics on hematology and biochemical parameters in mice. The Bangladesh Veterinarian 30(1): $20-24$

Saleem AM, Zanouny Al and Singer AM (2017): Growth performance, nutrients digestibility, and blood metabolites of lambs fed diets supplemented with probiotics during pre- and post-weaning period. Asian-Australas J Anim Sci. Apr; 30(4):523-530. Doi: 10.5713/ajas.16.0691. Epub 2016 Dec 17.

Sarah Adjei-Fremah, Kingsley Ekwemalor, Emmanuel K. Asiamah, Hamid Ismail, Salam Ibrahim and Mulumebet Worku(2017):Effect of probiotic supplementation on growth and global gene expression in dairy cows Pages 1-7 | Received 07 Apr 2016, Accepted 30 Jan , Published online: 28 Feb 2017.

Schroeder B, Winckler C, Failing K and Breves G.(2004):Studies on the time course of the effects of the probiotic yeast Saccharomyces boulardii on electrolyte transport in pig jejunum. Dig Aug; 49(78):1311-7

Segawa S, Wakita Y, and Hirata H, Watari J. (2008): Oral administration of heat-killed Lactobacillus brevis SBC8803 ameliorates alcoholic liver disease in ethanol-containing diet-fed C57BL/6N mice. Int J Food Microbiol; 128:371-7.

Shareef.AM and Dabbagh.ASA (2009): Effect of probiotic (saccharomyces cerevisiae) on performance of broiler chickens Iraqi .J.VeL Sci, 28:2829.

Sobhy M.Sallam, Ali M.Sallam and Samir A.Najadi (2014): Comparison of two products of direct-Fed microbial supplementation on the nutrient utilization and ruminal fermentation in sheep. Journal of Agricultural Science; Vol. 6, No. 3; ISSN 1916-9752 E-ISSN 19169760 Published by Canadian Center of Science and Education.

SPSS computer program version. 20 (2015).

Stoffregen, D .A, Wooster, G. A., Bustos, P.S. and Bowser, P.R. (1997): Multiple route and dose pharmacokinetics of ciprofloxacin in Juvenile Atlantic salmon J. Vet.Pharmacol. Ther; 20(2):111-123.

Strompfova V, Marcináková $\quad M$, Simonová $\quad M$, Gancarcíková $\quad S$, Jonecová Z, Sciranková L, Koscová J, Buleca V, Cobanová K, Lauková A(2006): Enterococcus faecium EK13--an enterocin a-producing strain with probiotic character and its effect in piglets. Anaerobe. Oct-Dec; 12(5-6):242-8. Epub 2006 Oct 27.

Vibhute V. M., R.R. Shelke, S.D. Chavan and S.P. Nage (2011): Effect of probiotics supplementation on the performance of lactating crossbred cows. Vet. World, Vol.4 (12):557-561.

Xing HC, Li LJ, Xu KJ, Shen T, Chen YB, Sheng JF (2006): Protective role of supplement with foreign Bifidobacterium and Lactobacillus in experimental hepatic ischemia-reperfusion injury. J Gastroenterol Hepatol; 21:647-56.

Zareshahneh.AR, AbdelahiARK.arnyab and Nikkhah(2007):Effect of different levels of bacterial probiotic on broilers performance and some of blood factors' Amic Oci Nat Res., 14:162-170. 